\title{
Well-Posed Two-Temperature Constitutive Equations for Stable Dense Fluid Shockwaves using Molecular Dynamics and Generalizations of Navier-Stokes-Fourier Continuum Mechanics
}

\author{
Wm. G. Hoover and Carol G. Hoover \\ Ruby Valley Research Institute \\ Highway Contract 60, Box 598, Ruby Valley 89833, NV USA
}

(Dated: April 19, 2022)

\begin{abstract}
Guided by molecular dynamics simulations, we generalize the Navier-Stokes-Fourier constitutive equations and the continuum motion equations to include both transverse and longitudinal temperatures. To do so we partition the contributions of the heat transfer, the work done, and the heat flux vector between the longitudinal and transverse temperatures. With shockwave boundary conditions time-dependent solutions of these equations converge to give stationary shockwave profiles. The profiles include anisotropic temperature and can be fitted to molecular dynamics results, demonstrating the utility and simplicity of a two-temperature description of far-from-equilibrium states.

PACS numbers:
\end{abstract}

Keywords: Temperature, Shockwaves, Molecular Dynamics, Computational Methods 


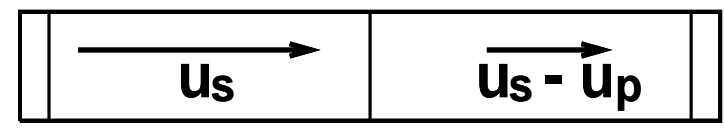

Figure 1: Schematic stationary shockwave. Cold fluid enters at the left cold boundary, with speed $u_{s}$; hot fluid leaves at the right hot boundary, with speed $u_{s}-u_{p}$. We choose a coordinate frame which moves leftward, at speed $u_{s}$ relative to the laboratory frame. The shockwave remains stationary in this coordinate frame.

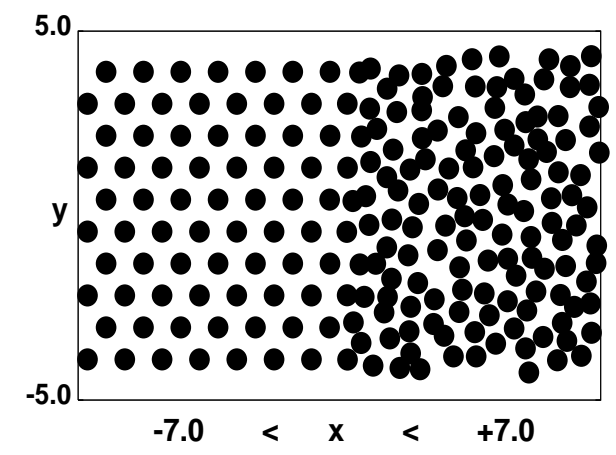

Figure 2: Stationary shockwave. Snapshot from a 10-row molecular dynamics simulation with a periodic height of $10 \sqrt{3 / 4}$. The simulations analyzed in the text are based on 80-row molecular dynamics with a periodic height of $80 \sqrt{3 / 4}$.

\section{INTRODUCTION}

A leftmoving piston, impacting a fluid with velocity $-u_{p}$, generates a leftmoving shockwave with velocity $-u_{s}$. Throughout this paper we analyze such a shockwave from the viewpoint of a coordinate system moving leftward, so as to keep pace with the shock. See Figures 1 and 2. In this special uniformly-translating coordinate frame the shockwave is stationary, simplifying theoretical analyses. One-dimensional stationary shockwaves $1-14$ provide a useful computational laboratory for the study of stationary far-from-equilibrium states. In such a shockwave a cold fluid is converted irreversibly to a hot one. As the fluid moves from left to right, in the shock-centered coordinate frame of the Figures, at speed $u(x)$, the $x$ coordinate increases; typically, the corresponding density, the longitudinal component of the pressure tensor, and the energy all increase too, in just such a way that the spatial structure of the wave is stationary:

$$
\begin{gathered}
\left\{u=\dot{x}, \dot{\rho}, \dot{P}_{x x}, \dot{e}\right\}>0 \\
(\partial u / \partial t)_{x}=0 ;(\partial \rho / \partial t)_{x}=0 ;\left(\partial P_{x x} / \partial t\right)_{x}=0 ;\left(\partial P_{y y} / \partial t\right)_{x}=0 ;(\partial e / \partial t)_{x}=0 .
\end{gathered}
$$


As the velocity decreases from its leftmost entrance value, $u(x \rightarrow-\infty)=u_{s}$, to its rightmost exit value, $u(x \rightarrow+\infty)=u_{s}-u_{p}$, the stationary nature of the wave requires that the fluxes of mass, momentum, and energy remain constant throughout:

$$
\begin{gathered}
(\rho u)_{x}=(\rho u)_{\mathrm{cold}}=(\rho u)_{\mathrm{hot}} ; \\
\left(P_{x x}+\rho u^{2}\right)_{x}=\left(P+\rho u^{2}\right)_{\mathrm{cold}}=\left(P+\rho u^{2}\right)_{\mathrm{hot}} ; \\
(\rho u)\left[\left(e+\left(P_{x x} / \rho\right)+\left(u^{2} / 2\right)\right]_{x}+Q_{x}=\right. \\
(\rho u)\left[e+(P / \rho)+\left(u^{2} / 2\right)\right]_{\mathrm{cold}}=(\rho u)\left[e+(P / \rho)+\left(u^{2} / 2\right)\right]_{\mathrm{hot}} .
\end{gathered}
$$

The notation here is conventional, with the pressure tensor $P$ and heat flux vector $Q$ assumed to be calculable from the density $\rho$, velocity $u$, energy $e$, and their gradients.

Temperature $11,12,15-17$ is our special interest in this work. Temperature is most simply and usefully defined as a velocity fluctuation, the "kinetic temperature":

$$
k T_{x x} \equiv m\left\langle(\dot{x}-\langle\dot{x}\rangle)^{2}\right\rangle ; k T_{y y} \equiv m\left\langle(\dot{y}-\langle\dot{y}\rangle)^{2}\right\rangle
$$

The angular brackets imply a local average. The velocities here are individual particle velocities, whose local average would be the hydrodynamic flow velocity $u$. Temperature is just the fluctuation about this average. It is evident that $T_{x x}$ and $T_{y y}$ can differ. In dilute-gas kinetic theory, the difference corresponds to a shear stress:

$$
\rho k\left(T_{x x}-T_{y y}\right) /(2 m)=\left(P_{x x}-P_{y y}\right) / 2 \quad[\text { Dilute Gas }]
$$

where $k$ is Boltzmann's constant and $m$ is the particle mass, which we choose equal to unity in what follows. In dense fluids there is no simple relationship between the two tensors so that special evolution equations for $T_{x x}$ and $T_{y y}$ need to be developed, as we do in Section III.

The cold fluid, initially moving to the right at the entrance velocity, or "shock velocity" $u_{s}$, is slowed by its encounter with the wave until it reaches its exit velocity $u_{s}-u_{p}$, where $u_{p}$ is the "piston velocity" or "particle velocity". In this irreversible deceleration the kinetic energy lost by the decelerating fluid is converted into additional hot fluid enthalpy

$$
\begin{aligned}
(H= & E+P V \leftrightarrow h=e+P v): \\
& h_{\mathrm{hot}}-h_{\mathrm{cold}}=[e+(P / \rho)]_{\mathrm{hot}}-[e+(P / \rho)]_{\mathrm{cold}}=\left[\rho_{\text {cold }} u_{s}^{2} / 2\right]-\left[\rho_{\mathrm{hot}}\left(u_{s}-u_{p}\right)^{2} / 2\right] .
\end{aligned}
$$



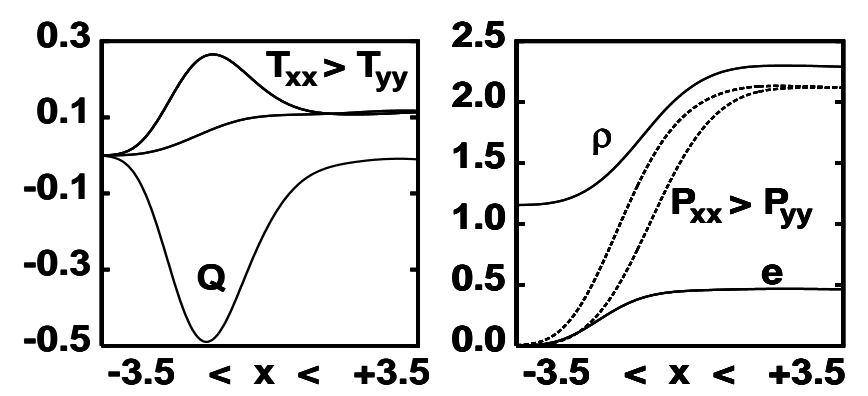

Figure 3: A snapshot spatial profile of a nominally steady one-dimensional shockwave from molecular dynamics, using a short-ranged repulsive potential. Spatial one-dimensional averages of the temperatures and heat flux (left) and the pressures, density, and energy (right) have been computed with Lucy's weight function using a range $h=3$. The cold zero-pressure, zerotemperature triangular lattice is compressed to twice the initial density $(\sqrt{4 / 3} \rightarrow 2 \sqrt{4 / 3})$ by the shockwave, just as in Figure 2.

The cold and hot boundary conditions enclosing the shock are equilibrium ones imposed far from the shockfront so that the small-system surface effects complicating the numberdependence of nonequilibrium systems are minimized. In implementing these ideas no arbitrary or artificial assumptions have to be made. All the observed phenomena follow from the assumed form for the interparticle forces. Figures 3, 4, and 5 show typical results from molecular dynamics, as is described in more detail in Section II. Notice that the rise in longitudinal temperature $T_{x x}$ can be much larger and can occur somewhat earlier $\frac{12}{2}$ than that of the transverse temperature $T_{y y}$.

In Section III we discuss the continuum mechanics of the same shockwave problem. Evidently any continuum formulation must first of all include the continuum conservation laws for mass, momentum, and energy:

$$
\begin{gathered}
\dot{\rho}=-\rho \nabla \cdot u ; \\
\rho \dot{u}=-\nabla \cdot P ; \\
\rho \dot{e}=-\nabla u: P-\nabla \cdot Q .
\end{gathered}
$$

Here the pressure tensor $P$ and heat flux vector $Q$ measure the momentum and energy fluxes in the local "comoving" (or "Lagrangian") coordinate frame moving with the mean velocity $u(x)$. Now the superior dot notation is used to indicate the time derivatives of 

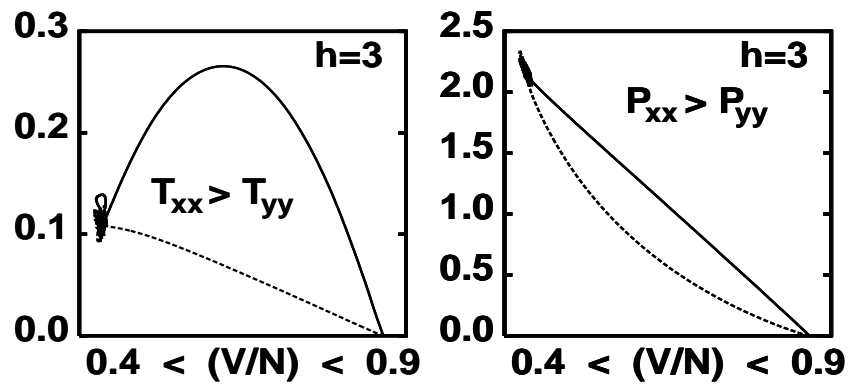

Figure 4: Volume dependence of the temperature tensor (left) and the pressure tensor (right) in the stationary shockwave of Figure 3, as calculated with molecular dynamics. Spatial averages have been computed with Lucy's weight function using a range $h=3$, as is discussed in Section II.
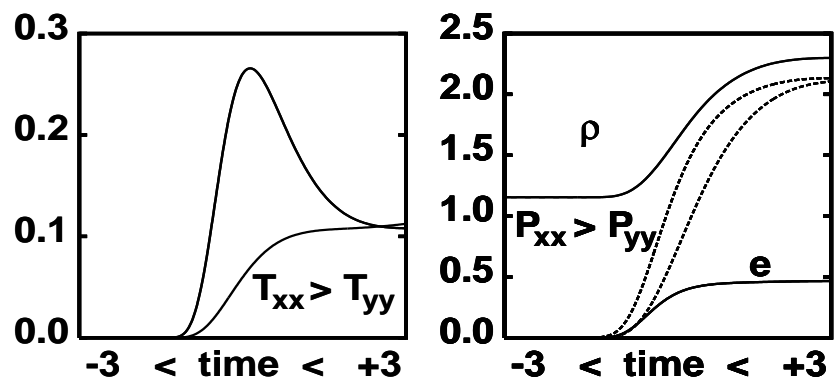

Figure 5: Stationary temporal profile for the one-dimensional shockwave of Figure 3, using a short-ranged repulsive potential. Spatial averages of the temperatures (left) and the pressures, density, and energy (right) have been computed with Lucy's weight function using a range $h=3$. The initial stress-free cold triangular lattice is compressed to twice the initial density by the shockwave, as in Figure 2. The time origin has been chosen, arbitrarily, close to the shockfront.

$\rho, u$, and $e$ following the motion at velocity $u$. In the continuum description these field variables are continuous differentiable functions of space and time so that the spatial averaging (necessary to an analysis of molecular dynamics data) is unnecessary.

The steady nature of the shock process makes it possible to use either space or time as an independent variable. On the average, the progress of a particle traveling through the shockwave follows from the integral of the flow velocity. To illustrate, consider again 

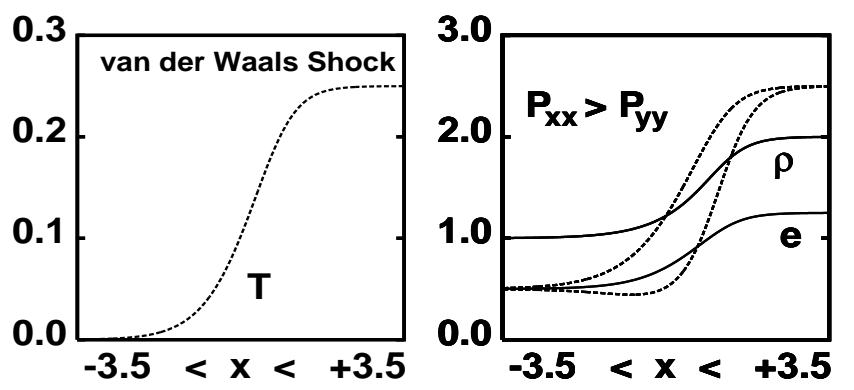

Figure 6: Stationary spatial profile for a one-dimensional shockwave according to the usual Navier-Stokes-Fourier equations for the model fluid: $P_{\text {eq }}=\rho e ; e=(\rho / 2)+k T$ with unit shear viscosity, zero bulk viscosity, and unit Fourier heat conductivity. Here the temperature $T$ (left) is a scalar, as in conventional continuum mechanics.

the molecular dynamics profiles shown in Figures 3, with space as the abscissa. Exactly the same profiles can alternatively be expressed with time as the abscissa, as in Figure 5. To change from space-based to time-based profiles requires use of the ratio $(d x / d t) \equiv u$ :

$$
\int_{0}^{t} d t^{\prime}=\int_{x_{0}}^{x} d x^{\prime} / u\left(x^{\prime}\right) ; t=0 \leftrightarrow x=x_{0} .
$$

where $u(x)$ is the hydrodynamic flow velocity. Thus all the spatial snapshots or equivalent temporal wave profiles catalog the sequence of time-ordered states through which the particles in a typical volume (initially at $x_{0}$ ) pass as they transit the shockwave.

Because the conventional Navier-Stokes-Fourier approach, illustrated in Figure 6, assumes a scalar temperature, $T=T_{x x}=T_{y y}$, several modifications of the continuum description need to be made to model the two-temperature results of Figures 3-5 found with molecular dynamics, with $T_{x x} \neq T_{y y}$. In Section III we describe simple modifications of the Navier-Stokes-Fourier constitutive and flow equations, along with a numerical method which converges nicely to give stationary shockwave profiles in the two-temperature case.

Section IV is reserved for a summary and our concluding remarks, including suggestionns for adapting our ideas to detailed two- and three-dimensional descriptions of the fluctuations in nonequilibrium systems. 


\section{RESULTS FROM MOLECULAR DYNAMICS}

The molecular dynamics simulations leading to our current results are all based on a very simple model two-dimensional system of unit-mass unit-radius particles interacting pairwise with a short-ranged normalized repulsive potential12,15.

$$
\phi(r<1)=(10 / \pi)(1-r)^{3} \rightarrow \int_{0}^{1} 2 \pi r d r \phi(r) \equiv 1 .
$$

The length and energy scales set by this potential correspond to the range and strength of the interparticle pair forces. The equilibrium properties for this potential can be approximated very roughly by a theoretical model (based on a random distribution of particles in space) resembling van der Waals' mean-field idea,

$$
P=\rho e ; e=(\rho / 2)+k T \text {. }
$$

$P, \rho, e$, and $T$ are the pressure, density, energy, and temperature. Though the models and language here all refer to systems in two space dimensions the same ideas can be applied equally well to three-dimensional systems.

We expect that the nonequilibrium properties for this model will likewise provide a simple interpretation. We are particularly interested here in generalizing the notion of temperature to the tensor case, $T_{x x} \neq T_{y y}$. The need for this generalization stems from the molecular dynamics shockwave simulations summarized in Figures 3, 4, and 5 .

Stationary shockwaves were obtained from molecular dynamics by matching the mass flux of a cold stress-free lattice $(\rho=\sqrt{4 / 3}$ and speed 1.930) to the mass flux of the hot fluid exiting at the righthand boundary (with $\rho=2 \sqrt{4 / 3}$ and speed 0.965):

$$
\rho u=\rho_{\text {cold }} u_{\text {cold }}=\rho_{\text {hot }} u_{\text {hot }}=1.93 \times \sqrt{4 / 3}=2.229
$$

With this choice for the shockwave speed $u_{s}=1.93$ and particle (or piston) speed $u_{p}=$ $u_{s} / 2$ the shockwave is stationary and corresponds to twofold compression, a "strong" shockwave ${ }^{12}$. The Mach number $M=u / c_{s}$ is not a useful description here as the sound speed $c_{s}$ vanishes in the cold state. The momentum and energy fluxes throughout the wave are equal to those of the initial cold lattice:

$$
\begin{gathered}
P_{x x}+\rho u^{2}=\sqrt{4 / 3}(1.93)^{2}=4.301 ; \\
\rho u\left[e+\left(P_{x x} / \rho\right)+\left(u^{2} / 2\right)\right]+Q_{x}=\sqrt{4 / 3}(1.93)^{3} / 2=4.151 .
\end{gathered}
$$


Spatial averages within the shockwave were calculated here using Lucy's weight function $12,13,15,16$,

$$
w_{\text {Lucy }}(|x|<h)=(5 / 4 h)\left[1-6 r^{2}+8 r^{3}-3 r^{4}\right] ; r \equiv|x| / h<1
$$

with a range equal to three times the range of the potential, $h=3$. The internal energy at a gridpoint coordinate $x$, for example, is computed as a ratio of sums:

$$
e(x)=\frac{\sum_{i} w\left(x-x_{i}\right) e_{i}}{\sum_{i} w\left(x-x_{i}\right)},
$$

where the energy of Particle $i$ is the sum of its kinetic energy relative to the local flow velocity $u(x)$ plus half its summed-up interaction energy with other nearby Particles $\{j\}$.

Consider now the results shown in Figures 3 and 4. The density, energy, and pressure agree roughly with the hyperbolic-tangent profiles derived by Landau and Lifshitz for a weak shockwave with constant transport coefficients ${ }^{3}$. Figure 4 shows the pressuretemperature-volume states through which the moving fluid travels. The Rayleigh Line, a straightline relation linking $P_{x x}$ and the volume, is necessarily satisfied and corresponds to the conservation of momentum. In marked contrast, the molecular dynamics temperature shows a strong maximum (as might be expected from the mixing of cold and hot Gaussian distributions suggested by Mott-Smith ${ }^{1}$ ) at the shockfront. Because the work done in compressing the fluid appears first in the longitudinal direction we expect that the rise in $T_{x x}$ precedes that of $T_{y y}$, as is confirmed in Figure 3. This thermal anisotropicity differs from the conventional textbook result and is the main motivation for our work on a two-temperature continuum description, detailed in the following Section.

\section{RESULTS FROM CONTINUUM MECHANICS}

\section{A. General Considerations}

Continuum models combine the universal conservation laws (mass, momentum, and energy) and the corresponding evolution equations (continuity, motion, and energy) with specific constitutive models. The constitutive models describe the pressure tensor and the heat flux vector for nonequilibrium systems. The usual Navier-Stokes assumptions, which we follow here for a two-dimensional fluid, are that the pressure tensor and heat flux vector respond linearly to velocity and temperature gradients:

$$
P=P^{\mathrm{eq}}-\lambda[\nabla \cdot u] I-\eta\left[\nabla u+\nabla u^{t}\right] ; \lambda \equiv \eta_{V}-\eta
$$




$$
Q=-\kappa \nabla T
$$

It needs to be emphasized that the choice of particular expansion variables, here $\nabla u$ and $\nabla T$, affects the solutions of nonlinear problems like shockwave structure. GarcíaColín and Green emphasized that the description of nonequilibrium continuum mechanics is ambiguous whenever the choice of "equilibrium" variables - energy or longitudinal temperature or transverse temperature in this case - is ambiguous $\frac{17}{2}$. The numerical value of a Taylor's series in the deviations from equilibrium, truncated after the first nonlinear term, is clearly sensitive to the choice of independent variable.

In the nonequilibrium pressure tensor the superscript ${ }^{t}$ indicates the transposed tensor and $I$ is the unit tensor:

$$
I_{11}=I_{22}=1 ; I_{12}=I_{21}=0
$$

$\eta$ is the shear viscosity, and $\lambda=\eta_{v}-\eta$ is defined by the bulk viscosity $\eta_{v}$. In the shockwave problem the pressure-tensor definitions give

$$
P_{x x}=P^{\mathrm{eq}}-\left(\eta_{v}+\eta\right) d u / d x ; P_{y y}=P^{\mathrm{eq}}-\left(\eta_{v}-\eta\right) d u / d x
$$

For a two-temperature continuum model it is necessary to formulate the "equilibrium pressure" $P^{\mathrm{eq}}$ as a function of the (nonequilibrium) energy, density, and the two temperatures. The viscosities and conductivity could likewise depend upon these state variables and $\kappa$ can be a tensor, as we show later, with an example.

When we define $T_{x x}$ and $T_{y y}$ as continuum state variables it becomes necessary for us to formulate constitutive relations for their evolution. The simplest such models begin by separating the energy into two parts: a density-dependent "cold curve" $e^{\text {cold }}(\rho)$ and an additional kinetic or "thermal" part, proportional to temperature:

$$
e \equiv e^{\text {cold }}(\rho)+e^{\text {thermal }}\left(T_{x x}, T_{y y}\right)=e^{\text {cold }}+(c k)\left(T_{x x}+T_{y y}\right)
$$

where $c k$ is a scalar heat capacity. The functional form of the cold curve produces a corresponding contribution to the pressure:

$$
P^{\text {cold }}=-d e^{\text {cold }} / d(V / N)=\rho^{2} d e^{\text {cold }} / d \rho
$$

Grüneisen's $\gamma$ defines a corresponding thermal pressure:

$$
P^{\text {thermal }}=\gamma \rho e^{\text {thermal }} .
$$


The viscous part of the pressure tensor is Newtonian:

$$
P^{\text {viscous }}=-\lambda \nabla \cdot u I-\eta\left(\nabla u+\nabla u^{t}\right) .
$$

The thermal and viscous parts of the First-Law energy change are then apportioned between the $x$ and $y$ directions so as to be consistent with overall energy conservation:

$$
\begin{gathered}
\dot{e}^{\text {thermal }}=\dot{e}-\dot{e}^{\text {cold }}(\rho)=c k \dot{T}_{x x}+c k \dot{T}_{y y} ; \\
\rho c k \dot{T}_{x x}=-\alpha \nabla u:\left(P-I P^{\text {cold }}\right)-\beta \nabla \cdot Q+\rho c k\left(T_{y y}-T_{x x}\right) / \tau ; \\
\rho c k \dot{T}_{y y}=(\alpha-1) \nabla u:\left(P-I P^{\text {cold }}\right)+(\beta-1) \nabla \cdot Q+\rho c k\left(T_{x x}-T_{y y}\right) / \tau .
\end{gathered}
$$

The thermal relaxation time $\tau$ has been introduced in the evolution equations to guarantee thermal equilibrium far from the shockwave:

$$
K_{x}=K_{y} \leftrightarrow T_{x x}=T_{y y}=T^{\mathrm{eq}}
$$

In what follows we consider two models for the cold curve and the heat capacity. First, a weak repulsive pair force suggests implementing a "van der Waals model":

$$
e^{\text {cold }}=(\rho / 2) ; e^{\text {thermal }}=k\left(T_{x x}+T_{y y}\right) / 2 ; P^{\text {eq }}=\rho e
$$

Second, a triangular-lattice-based model, based on Grüneisen's ideas, uses the nearestneighbor static lattice energy and pressure corresponding to the pair potential evaluated at the nearest-neighbor lattice spacing $r, \phi=(10 / \pi)(1-r)^{3}$ :

$$
\begin{gathered}
e^{\text {cold }}=(30 / \pi)(1-r)^{3} ; p^{\text {cold }}(V / N)=(45 / \pi) r(1-r)^{2} ; \\
r=\sqrt{V / V_{0}} ; V_{0}=\sqrt{3 / 4} N .
\end{gathered}
$$

The corresponding equilibrium equation of state separates the energy and pressure into "cold" and "thermal" parts:

$$
e^{\mathrm{eq}}=e^{\mathrm{cold}}+e^{\text {thermal }} ; P^{\mathrm{eq}}=P^{\text {cold }}+\rho \gamma e^{\text {thermal }},
$$

with $\gamma$ chosen so as to roughly reproduce equation of state data from molecular dynamics. Let us next apply these two simple cold-curve models to the shockwave problem. 


\section{B. Potential plus Kinetic van der Waals Models}

First consider an arbitrary, but simple and natural, choice:

$$
\begin{gathered}
P^{\mathrm{eq}}=\rho e ; e^{\mathrm{eq}}=e^{\text {cold }}+e^{\text {thermal }}=\left(\rho+k T_{x x}+k T_{y y}\right) / 2 . \\
P^{\text {cold }}=\rho e^{\text {cold }}=\rho^{2} / 2,
\end{gathered}
$$

with an initial density of unity and an initial temperature of zero. Twofold compression of the cold van der Waals fluid gives the following solution relating the initial and final equilibrium states:

$$
\rho: 1 \rightarrow 2 ; u: 2 \rightarrow 1 ; T: 0 \rightarrow 1 / 4 ; e: 1 / 2 \rightarrow 5 / 4 ; P: 1 / 2 \rightarrow 5 / 2 .
$$

The mass, momentum, and energy fluxes connecting these states must be constant throughout the profile:

$$
\rho u=2 ; P_{x x}+\rho u^{2}=9 / 2 ; \rho u\left[e+\left(P_{x x} / \rho\right)+\left(u^{2} / 2\right)\right]+Q_{x}=6 .
$$

Consider the most extreme anisotropic situation consistent with energy conservation, in which all the work done and heat transfered are associated with thermal change in the $x$ direction. The thermal relaxation time $\tau$, here chosen equal to unity, guarantees that the $x$ and $y$ temperatures equilibrate in a time of order $\tau$ :

$$
\begin{gathered}
\dot{e}^{\text {thermal }}=\dot{e}-\dot{e}^{\text {cold }}(\rho)=(k / 2)\left(\dot{T}_{x x}+\dot{T}_{y y}\right) ; \\
\rho(k / 2) \dot{T}_{x x}=-\nabla u:\left(P-I P^{\text {cold }}\right)-\nabla \cdot Q+\rho(k / 2)\left(T_{y y}-T_{x x}\right) / \tau ; \\
\rho(k / 2) \dot{T}_{y y}=\rho(k / 2)\left(T_{x x}-T_{y y}\right) / \tau ; \tau=1 .
\end{gathered}
$$

Two solutions of these equations appear in Figures 7 and 8. For both of them we chose a shear viscosity of unity and a vanishing bulk viscosity:

$$
P_{x x}=P^{\mathrm{eq}}-d u / d x ; P_{y y}=P^{\mathrm{eq}}+d u / d x
$$

The heat flux vector requires that an additional choice be made for its response to the gradients of $T_{x x}$ and $T_{y y}$. We compare two choices in Figures 7 and 8 . For both of them the overall conductivity is unity, but the heat flux responds differently to the two components of $\nabla T$ :

$$
Q_{x}=-\kappa \nabla T_{y y}=-\nabla T_{y y} \quad[\text { Choice } 1] .
$$




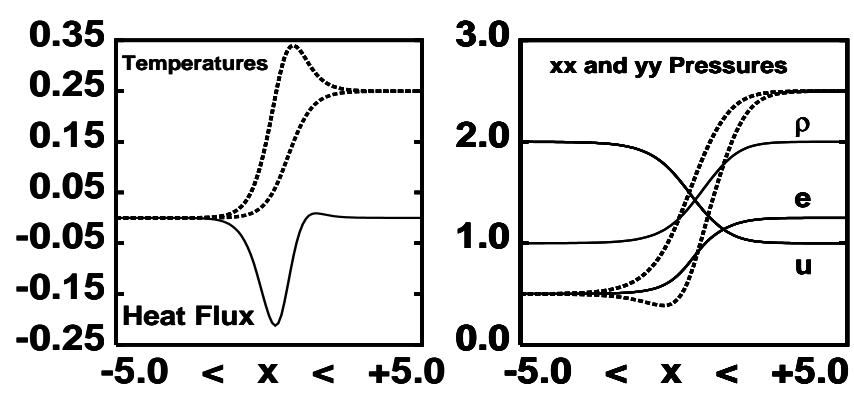

Figure 7: Typical solution of the generalized Navier-Stokes-Fourier equations for the van der Waals model with both heat and work contributing to $T_{x x}$ and with the heat flux responding only to the gradient of $T_{y y}$. The shear viscosity, heat conductivity, heat capacity, and thermal relaxation times are all taken equal to unity.

$$
Q_{x}=-\kappa\left(\nabla T_{x x}+\nabla T_{y y}\right) / 2=-\left(\nabla T_{x x}+\nabla T_{y y}\right) / 2 \quad[\text { Choice 2] }
$$

It is good fortune that the shockwave equations we summarize here are relatively easy to solve numerically. The usual numerical method is the "backward Euler" scheme 2 . One starts near the "hot" boundary and integrates backward, using a first-order difference scheme. That approach fails here, due to the temperature relaxation terms, which are exponentially unstable in the time-reversed case. An integration forward in time is required in the presence of relaxation. A successful "staggered-grid" (two separate spatial grids) algorithm results if the density $\rho_{c}$ is defined at cell centers and energy, temperature, and pressure are defined at the nodes which bound the cells $\frac{18,19}{19}$. This algorithm follows the dynamics correctly and converges nicely to the stationary profiles shown in Figures 7 and 8. A computational mesh spacing of $d x=0.1$ is sufficient, using the second-order spatial differencing scheme outlined in References 18 and 19 with fourth-order Runge-Kutta time integration.

In the early days of shockwave modeling this computational simplicity was by no means apparent, so that there is an abundant literature on the stability of numerical methods for the shockwave problem ${ }^{2}$. Now, in the early days of tensor-temperature models, the main challenge is to develop well-posed constitutive equations consistent with both the conservation laws and the empirical results from molecular dynamics.

Interesting aspects of both solutions are (i) the minimum in $P_{y y}(x)$, which suggests the need for bulk viscosity in modeling molecular dynamics results, and (ii) the pronounced 

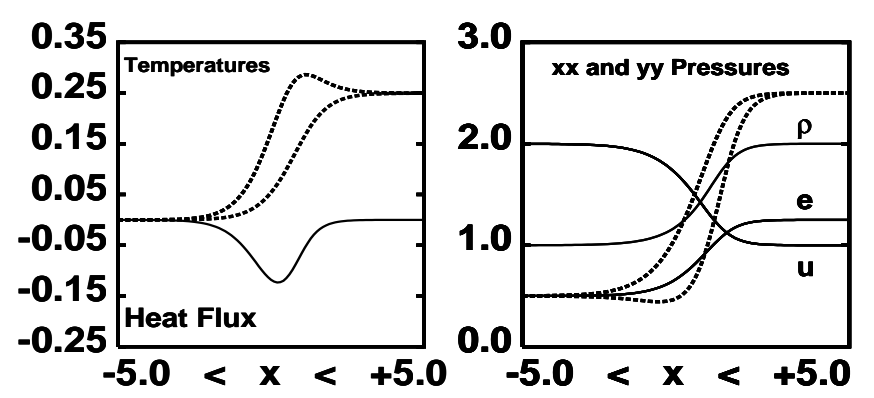

Figure 8: Typical solution of the generalized Navier-Stokes-Fourier equations for the van der Waals model with both heat and work contributing to $T_{x x}$ and with the heat flux responding equally to the gradients of both $T_{x x}$ and $T_{y y}$. The shear viscosity, heat conductivity, heat capacity, and thermal relaxation times are all taken equal to unity.

maximum in $T_{x x}(x)$, leading the response of $T_{y y}$ and roughly equal in magnitude to that found in the dynamical results of Section II.

The physical ideas incorporated in this simplest approach are four: (i) the pressure and the work done can usefully be separated into a "cold" part and a "thermal" part; (ii) the heat flux $Q$ responds to a linear combination of the temperature gradients $\nabla T_{x x}$ and $\nabla T_{y y}$ in the usual way, supplemented by (iii) the thermal relaxation of the thermal anisotropicity, and (iv) separate linear combinations of the work done and heat absorbed contribute to $T_{x x}$ and $T_{y y}$ throughout the shock compression process.

Here the total pressure, $P=P^{\Phi}+P^{K}$, contains potential and kinetic components, measurable separately with molecular dynamics. These extensions of the Navier-Stokes approach closely parallel the relaxation-time treatments of strong ideal-gas shockwaves carried out by $\mathrm{Xu}$, Josyula, Holian, and Mareschal 11,14 . Our more general approach necessarily differs from theirs by allowing for contributions from the potential energy to temperature changes and the transfer of heat. The pressure profiles shown in Figures 7 and 8 also indicate the need for bulk viscosity, in that the molecular dynamics results show a monotone-increasing $P_{y y}$, in contrast to the distinct minimum found here in the absence of bulk viscosity. We turn next to a slightly more sophisticated model, an extension of Grüneisen's equilibrium equation of state. 


\section{Cold plus Thermal Grüneisen Models}

For gases, where the pressure and temperature tensors are proportional to one another, a systematic expansion of the Boltzmann equation can be, and has been, tried $10,11,14,17$. $\mathrm{Xu}$ and Josyula ${ }^{11}$ as well as Holian and Mareschal ${ }^{14}$ developed solutions of generalized relaxation-time Boltzmann equations for the shockwave problem. For dense fluids only Enskog's hard-sphere-based theory is available. More flexible empirical models need to be developed for dense-fluid shockwaves. A trial set of two-temperature evolution equations, the simplest plausible set generalizing the van der Waals model above, makes use of Grüneisen's "cold curve" representation of the energy and pressure to define "thermal" contributions. These thermal parts include both the effects of thermal agitation (heat and temperature) and of mechanical distortion (work, through compression with viscous deformation):

$$
E=\Phi^{\text {cold }}+E^{\text {thermal }} ; P_{(x x \text { and } y y)}=P^{\text {cold }}+P^{\text {thermal }}+P^{\text {viscous }} .
$$

For the molecular dynamics simulations discussed in Section II the cold parts of the pressure and energy, as well as their time dependence, are naturally defined by imagining a perfect static triangular lattice of particles:

$$
\begin{gathered}
E^{\text {cold }} / N=e^{\text {cold }}=(30 / \pi)(1-r)^{3} ; P^{\text {cold }} V / N=-\left(d E^{\text {cold }} / d V\right)=(45 / \pi) r(1-r)^{2} . \\
\rho \dot{e}^{\text {cold }}=-\nabla u: P^{\text {cold }} .
\end{gathered}
$$

Here $r$ is the separation of the six nearest neighbors in a cold triangular lattice, so that $\rho=\sqrt{4 / 3} / r^{2}$.

Just as in the equilibrium Grüneisen model the thermal energy and the nonviscous part of the thermal pressure are taken to be proportional to temperature:

$$
e^{\text {thermal }}=c\left(K_{x}+K_{y}\right) / N ; P^{\text {thermal }}=\gamma \rho e^{\text {thermal }}
$$

where $\gamma$ is Grüneisen's constant and $c k$ is a heat capacity.

The Krook-Boltzmann relaxation terms, with relaxation time $\tau$, are the simplest means for guaranteeing thermal equilibrium, with the two temperatures approaching one another far from the shockfront.

Because molecular dynamics simulations indicate that temperature becomes a tensor in strong shockwaves, a tentative two-temperature formulation can be based on separating 
the internal energy and the pressure into the three components suggested by classical statistical mechanics, including Newtonian shear and bulk viscosities:

$$
\begin{gathered}
E=N e=\Phi^{\text {cold }}+\Phi^{\text {thermal }}+K_{x}+K_{y} ; \\
P_{x x}=P_{e q}-\left(\eta+\eta_{V}\right) d u / d x ; P_{y y}=P_{e q}+\left(\eta-\eta_{v}\right) d u / d x ; \\
P_{e q}=\rho\left[\phi^{\text {cold }}+\gamma c k T_{x x}\right] \text { or } \rho\left[\phi^{\text {cold }}+\gamma c k T_{y y}\right] \text { or } \rho\left[\phi^{\text {cold }}+\gamma c k\left(T_{x x}+T_{y y}\right) / 2\right] ; \\
e^{\text {thermal }}=\phi^{\text {thermal }}+(k / 2)\left(T_{x x}+T_{y y}\right)=c k\left(T_{x x}+T_{y y}\right) .
\end{gathered}
$$

The sum of the three energy evolution equations just given is designed to reproduce the usual First Law energy equation,

$$
\dot{E}=\dot{E}_{Q}-\dot{E}_{W}
$$

where $\dot{E}_{Q}$ and $\dot{E}_{W}$ are the comoving rates at which heat enters the fluid and at which the fluid performs work on its surroundings. The constitutive relations for $P$ and $Q$ must also be given. For a two-dimensional Newtonian fluid with shear viscosity $\eta$ and bulk viscosity $\eta_{v}$ we have

$$
P_{x x}=P_{\mathrm{eq}}-\left(\eta+\eta_{v}\right) d u / d x ; P_{y y}=P_{\mathrm{eq}}+\left(\eta-\eta_{v}\right) d u / d x
$$

The heat flux is given by a generalization of Fourier's law, with independent contributions from $\nabla T_{x x}$ and $\nabla T_{y y}$.

Additional generalizations of this approach can be developed as needed to describe results from simulations. It is only required that any such model satisfy energy conservation and reduce to the Navier-Stokes-Fourier model in the weak-shock limit. To illustrate the possibilities, compare the molecular dynamics results of Figure 3 to the model calculations of Figure 9. In Figure 9 the relaxation time has been increased to 3, the heat capacity doubled, to $c k=2 k$, and the heat conductivity set equal to 6 so as to better match the empirical results of molecular dynamics. The value of Grüneisen's $\gamma$ is 0.3 , and the bulk and shear viscosities are both equal to unity. The results from these choices (which are by no means optimized) resemble the shockwave profiles obtained with molecular dynamics.

\section{CONCLUSIONS AND PROBLEMS FOR THE FUTURE}

We have shown here that it is relatively easy to model the thermal anisotropicity found in atomistic simulations of strong shockwaves. Thermal relaxation, bulk viscosity, and 

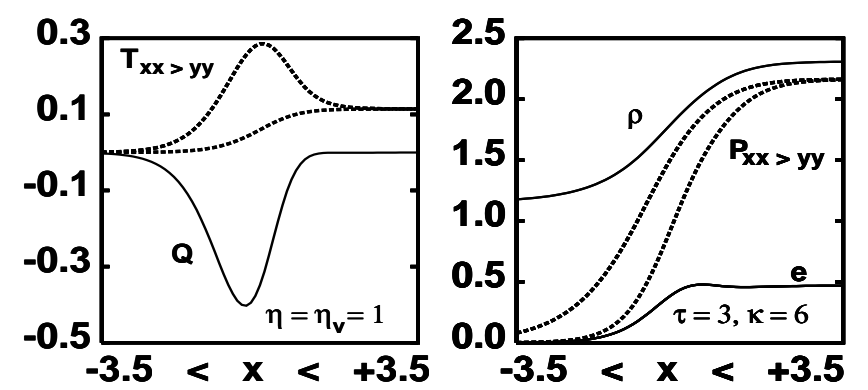

Figure 9: Solution of the generalized Navier-Stokes-Fourier equations with both heat and work contributing solely to $T_{x x}$ and with the heat flux $Q=-\kappa\left(\nabla T_{x x}+7 \nabla T_{y y}\right) / 8$. The shear viscosity, bulk viscosity, heat conductivity, and thermal relaxation times are respectively 1, 1, 6, and 3 . Grüneisen's $\gamma$ is 0.3 and $c k=2 k$.

Grüneisen equations of state are useful components of a kinetic shockwave model. By apportioning the longitudinal and transverse thermal portions of the work, heat, and heat flux vector a variety of useful models can be developed and used to reproduce results from simulations. A forward-in-time fourth-order Runge-Kutta (as opposed to backward Euler) integration of the cell and nodal motion equations results in accurate and stable continuum dynamics.

One of the recent observations from molecular dynamics is that the stress and heat flux lag somewhat behind the strainrate and the temperature gradient $\frac{12}{2}$. It is desirable that models be generalized to reflect these lags. Some study of time-delayed differential equations is necessary to model this phenomenon.

A significant goal is the extension of these same ideas to the fluctuating stress and heat flows of two and three dimensional fluids. A comparison of results from molecular dynamics with those from two and three-dimensional two-temperature continuum simulations should provide useful tools for describing fluctuations within the overall one-dimensional flows.

These results show that even far-from-equilibrium shocks can be treated in a semiquantitative way by relating the tensor parts of the energy flows to one another in a relatively simple way. An intriguing result of some model calculations is the stable reversal of the direction of the heat flux vector. Though this reversal seems unphysical, there is no difficulty in obtaining stable numerical profiles which include flux reversal. 


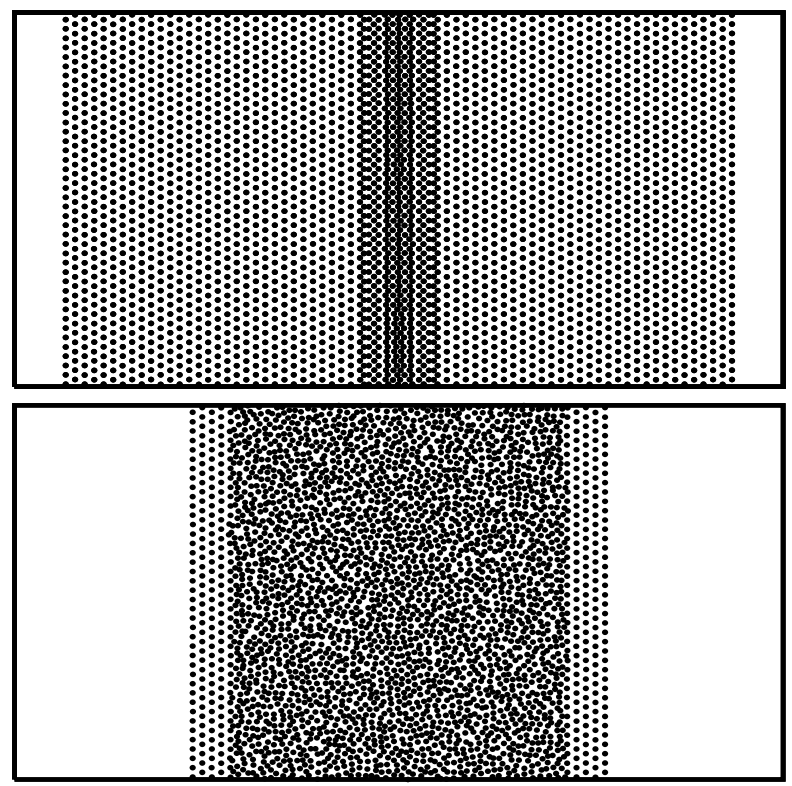

Figure 10: Two snapshots of the collision of two 1600-particle slabs (periodic in the $y$ direction, with height 20 and initial width $20 \sqrt{3 / 4}$. The initial velocities, $u_{p}= \pm 0.965$ give twofold shock compression, followed by a nearly isentropic free expansion at the free surfaces.

The thermodynamic irreversibility of the shockwave process has an interest independent of the definition of temperature and is worth futher study. The shock process itself obeys purely Hamiltonian mechanics, and Liouville's Theorem ${ }^{20}$. Even so, by using Levesque and Verlet's integer version of the leapfrog algorithm $\underline{21}$ the entire shockwave dynamics can be precisely reversed, to the very last bit. The apparent paradox, a perfectly time-reversible but thermodynamically irreversible process, can most clearly be illustrated by simulating the (inelastic) collision of two zero-pressure blocks of fluid. The collision of the blocks, with velocities $\pm u_{p}$ generates two shockwaves, with velocities $\pm\left(u_{s}-u_{p}\right)$. Two snapshots from such a simulation are shown in Figure 10.

\section{ACKNOWLEDGMENTS}

We appreciate stimulating comments from several colleagues: Paco Uribe, Vitaly Kuzkin, Howard Brenner, Michel Mareschal, Krzysztof Wojciechowski, and Jim Lutsko. 
Brad Holian and Michel Mareschal have provided continuing inspiration, through their emails and seminal publications.

1 H. M. Mott-Smith, "The Solution of the Boltzmann Equation for a Shockwave", Physical Review 82, 885-892 (1951).

2 D. Gilbarg and D. Paolucci, "The Structure of Shockwaves in the Continuum Theory of Fluids", Journal of Rational Mechanics Analysis 2, 617-642 (1953).

3 L. D. Landau and E. M. Lifshitz, Fluid Mechanics (Pergamon, Oxford, 1959). Chapter IX is devoted to shockwaves.

4 R. E. Duff, W. H. Gust, E. B. Royce, M. Ross, A. C. Mitchell, R. N Keeler, and W. G. Hoover, "Shockwave Studies in Condensed Matter", pp. 397-406 in Behavior of Dense Media under High Dynamic Pressures (Gordon and Breach, New York, 1968).

5 V. Y. Klimenko and A. N. Dremin, "Structure of Shockwave Front in a Liquid", pages 79-83 in Detonatsiya, Chernogolovka (Akademia Nauk, Moscow, 1978).

6 W. G. Hoover, "Structure of a Shockwave Front in a Liquid", Physical Review Letters 42, 1531-1534 (1979).

7 B. L. Holian, W. G. Hoover, B. Moran, and G. K. Straub, "Shockwave Structure via Nonequilibrium Molecular Dynamics and Navier-Stokes Continuum Mechanics", Physical Review A 22, 2798-2808 (1980).

8 B. L. Holian, "Modeling Shockwave Deformation via Molecular Dynamics", Physical Review A 37, 2562-2568 (1988).

9 O. Kum, Wm. G. Hoover, and C. G. Hoover, "Temperature Maxima in Stable TwoDimensional Shockwaves", Physical Review E 56, 462-465 (1997).

10 F. J. Uribe, R. M. Velasco, and L. S. García-Colín, "Two Kinetic Temperature Description for Shock Waves", Physical Review E 58, 3209-3222 (1998).

11 K. Xu and E. Josyula, "Multiple Translational Temperature Model and its Shock Structure Solution", Physical Review E 71, 056308 (2005).

12 Wm. G. Hoover and C. G. Hoover, "Tensor Temperature and Shockwave Stability in a Strong Two-Dimensional Shockwave", Physical Review E 80, 011128 (2009).

13 Wm. G. Hoover and C. G. Hoover, "Shockwaves and Local Hydrodynamics; Failure of the 
Navier-Stokes Equations", Condensed Matter arXiv:0909.2882.

14 B. L. Holian and M. Mareschal, "A New Heat-Flow Equation Motivated by the Ideal-Gas Shockwave", Physical Review E (submitted, 2009).

15 Wm. G. Hoover and C. G. Hoover, "Nonlinear Stresses and Temperatures in Transient Adiabatic and Shear Flows via Nonequilibrium Molecular Dynamics: Three Definitions of Temperature", Physical Review E 79, 046705 (2009).

16 Wm. G. Hoover, C. G. Hoover, and J. F. Lutsko, "Microscopic and Macroscopic Stress with Gravitational and Rotational Forces", Physical Review E 79, 036709 (2009).

17 L. S. García-Colín and M. S. Green, "Definition of Temperature in the Kinetic Theory of Dense Gases", Physical Review 150, 153-158 (1966).

18 A. L. Garcia, M. M. Mansour, G. C. Lie, and E. Clementi, "Numerical Integration of the Fluctuating Hydrodynamic Equations", Journal of Statistical Physics 47, 209-228 (1987).

19 A. Puhl, M. M. Mansour, and M. Mareschal, "Quantitative Comparaison of Molecular Dynamics with Hydrodynamics in Rayleigh-Bénard systems", Physical Review A 40 1999-2012, (1989).

20 Wm. G. Hoover, "Liouville's Theorems, Gibbs' Entropy, and Multifractal Distributions for Nonequilibrium Steady States", Journal of Chemical Physics 109, 4164-4170 (1998).

21 O. Kum and W. G. Hoover, "Time-Reversible Continuum Mechanics", Journal of Statistical Physics 76, 1075-1081 (1994). 